05

\title{
Зондовая микроскопия и электронно-транспортные свойства тонких эпитаксиальных пленок Мо на сапфире
}

\author{
(ㄱ Л.А. Фомин, ${ }^{1}$ И.В. Маликов, ${ }^{1}$ В.А. Березин, ${ }^{1}$ А.В. Черных, ${ }^{1}$ А.Б. Логинов, ${ }^{2}$ Б.А. Логинов ${ }^{3}$ \\ ${ }^{1}$ Институт проблем технологии микроэлектроники и особо чистых материалов РАН, \\ 142432 Черноголовка, Московская обл., Россия \\ ${ }^{2}$ Московский государственный университет им. М.В. Ломоносова, \\ 119991 Москва, Россия \\ ${ }^{3}$ Национальный исследовательский университет МИЭТ, \\ 124498 Москва, Россия \\ e-mail: fomin@iptm.ru
}

Поступило в Редакцию 1 апреля 2020 г.

В окончательной редакции 1 апреля 2020 г.

Принято к публикации 1 апреля 2020 г.

Проведены исследования поверхности и электронно-транспортных свойств эпитаксиальных тонких пленок молибдена. Экспериментальные результаты сравнивались с известными квантовыми моделями влияния рельефа поверхности пленок на их сопротивление.

Ключевые слова: эпитаксиальные пленки, тугоплавкие металлы, межсоединения, шероховатая поверхность, атомно-силовая микроскопия.

DOI: $10.21883 / J T F .2020 .11 .49970 .110-20$

\section{Введение}

В современной кремниевой технологии микроэлектроники в качестве межсоединений. выступают металлические пленочные поликристаллические проводники. Было показано [1], что увеличение удельного сопротивления проводящей линии будет сильно ограничивать длину межсоединения и отрицательно влиять на характеристики интегральных микросхем. В международном плане по развитию полупроводниковой технологии (ITRS) проблема увеличения сопротивления при уменьшении размеров металлических подводящих соединений была названа „Большим вызовом“.

Традиционно применяемые в настоящее время для металлизации в микроэлектронике алюминий и медь с уменьшением размеров элементов и возрастанием плотности тока в них перестают удовлетворять современным требованиям. Низкие температуры плавления этих металлов ограничивают их применение, поскольку характерные процессы деградации, приводящие к отказам активных и пассивных элементов интегральных схем, активируются с ростом отношения рабочей температуры к температуре плавления.

Одним из методов решения указанных проблем является поиск альтернативных материалов для технологии интегральных схем. При этом с точки зрения надежности очевидным преимуществом обладают системы, использующие тугоплавкие металлы. Например, вольфрам в сравнении с алюминием при относительно низком удельном сопротивлении характеризуется высокой температурой взаимодействия с кремнием и низким значением коэффициента линейного расширения. По этой причине вольфрам достаточно давно рассматривался как материал для шин металлизации интегральных схем, работающих при высоких температурах и плотностях тока [2].

Основным механизмом возникновения сопротивления в металлах при комнатной температуре является рассеяние электронов проводимости на фононах (колебаниях решетки). Электроны также могут рассеиваться на примесях, плоских и линейных дефектах в кристаллах и на внешних поверхностях проводника. По мере того, как геометрические размеры проводника уменьшаются и становятся сравнимыми с длиной свободного пробега электронов или даже меньше, вклад в сопротивление, обусловленный рассеянием электронов на внешних поверхностях проводника, возрастает.

В связи с развитием суб- $10 \mathrm{~nm}$ электроники при уменьшении линейных размеров элементов металлических материалов до величин, сравнимых с длиной пробега электронов, качество поверхностей начинает иметь важное значение в проводимости и транспортных эффектах. Сопротивление медного проводника шириной $10 \mathrm{~nm}$ возрастает более чем на порядок [3]. Непосредственной причиной этого является рассеяние электронов проводимости на поверхности проводника, а также границах зерен, обычно описываемое классическими моделями Фукса-Зондгеймера $[4,5]$ и Майадеса и Шацкеса [6,7] на основе решения уравнения переноса Больцмана. В модели Фукса-Зондгеймера задается коэффициент зеркальности $p$, который есть вероятность того, что электроны отражаются от поверхности зеркально, а $1-p$ есть вероятность диффузного рассеяния электронов. Этот коэффициент является единственным параметром поверхности в данной модели. При зеркальном отражении электроны проводимости не теряют энергию, 
таким образом, оно не приводит к увеличению сопротивления. Для больших размеров проводника эта модель предсказывает для удельного сопротивления обратную пропорциональность от размеров, что также подтверждается экспериментальными измерениями. Однако с уменьшением размеров большую роль начинают играть квантовые эффекты [8-12], которые в данной модели игнорируются, так как в нее не входят длина волны электрона, угол падения и шероховатость границы раздела. Позже была предложена [13] альтернативная модель рассеяния на поверхности, в которой коэффициент зеркальности зависит от угла подлета (угла скольжения) электронов к поверхности, а также от ее шероховатости. Согласно этой модели, увеличение удельного сопротивления проводника в основном обусловлено рассеянием электронов, налетающих на шероховатую поверхность под большими углами скольжения. Малоугловые электроны, летящие параллельно поверхности, „шунтируют“ сопротивление пленки. При малых углах подлета вероятность диффузного рассеяния $1-p$ пропорциональна углу подлета с коэффициентом пропорциональности $Q$, который определяет интенсивность рассеяния электронов из-за шероховатости поверхности. Однако для малых размеров проводника эта модель также не годится, так как даже в условиях правомерности квазиклассического приближения для корректного описания отражения электронов от поверхности и выводу граничных условий для функции распределения в уравнении Больцмана необходимо привлечение квантовых моделей [14-17]. Разработанные подходы, например, для гелиевых температур, позволят применить и учитывать их и для комнатной температуры.

Экспериментальные данные по размерным эффектам для пленок металлов сверхмалых толщин крайне ограничены. По всей видимости, это связано со сложностью получения однородных сплошных пленок сверхмалых толщин и измерений их характеристик. Требуется выращивание сплошных пленок с высокой степенью однородности в диапазоне толщин $0.5-10 \mathrm{~nm}$. Имеются экспериментальные данные о пленках $\mathrm{Pt}$, в которых были обнаружены осцилляции удельного сопротивления в зависимости от толщины. Некоторые интересные результаты были представлены в работе $[18,19]$ для пленок $\mathrm{CoSi}_{2}$ в диапазоне толщин 6-100 nm. Было найдено, что эти пленки являются металлическими и их остаточная длина свободного пробега составляла около $100 \mathrm{~nm}$. В работах [20,21] проведены измерения поверхности тонких $(70 \mathrm{~nm})$ пленок золота, нанесенных на слюду в сверхвысоком вакууме. Из измерений были получены автокорреляционные функции для сканов поверхности размерами $20 \times 20 \mathrm{~nm}$ и определены статистические параметры шероховатой поверхности, такие как среднеквадратичная флуктуация и корреляционная длина. Зависимости проводимости пленок от температуры и толщины сравнивались с теорией из работ $[15,16]$. Толщинные зависимости проводимости пленок золота на слюде были взяты из работы [22]. Значения толщин составляли от 35 до $126 \mathrm{~nm}$. Полученные экспериментальные результаты достаточно хорошо согласовывались с теорией. В наших более ранних работах $[12,23,24]$ исследовалась поверхность и электронно-транспортные свойства пленок $\mathrm{W}, \mathrm{Ni}$ и Мо. Однако шероховатость и электронно-транспортные свойства для сверхмалых толщин не были исследованы. В настоящей работе проведены дополнительные исследования пленок Мо для сверхмалых толщин.

В настоящей работе была использована разработанная нами модель влияния шероховатости поверхности пленок металлов на их электронно-транспортные свойства. Изготовлены эпитаксиальные тонкие пленки молибдена по ранее разработанной технологии. Проведены измерения образцов методами атомно-силовой микроскопии (ACM) и сканирующей туннельной микроскопии (СТМ), направленные на исследование статистических свойств поверхности.

\section{1. Теоретическая модель}

Рассмотрим модель транспорта электронов по волноводу с шероховатой границей раздела с учетом размерного квантования. Будем считать, что электрон движется в прямоугольной потенциальной яме с бесконечно высокими стенками по оси $z$, в то время как движение в направлениях $x$ и $y$ свободное с волновым двумерным вектором $k$. Шероховатость поверхности изменяет ширину потенциальной ямы и ее положение относительно начала отсчета по оси $z$, входя тем самым в оператор потенциальной энергии $U(z(r))$, где $r=(x, y)-$ двумерный вектор в плоскости пленки. Таким образом, стационарное уравнение Шредингера записывается в виде

$$
-\frac{\hbar^{2}}{2 m} \nabla^{2} \psi+U(z(r)) \psi=E \psi .
$$

Шероховатую границу общего вида можно свести к гладкой с помощью преобразований сдвига и растяжения. Оператор сдвига и его обратный оператор имеют вид:

$$
\begin{gathered}
S_{h}=\delta\left(z_{1}+h\left(\mathbf{r}_{1}\right)-z_{2}\right) \delta\left(\mathbf{r}_{1}-\mathbf{r}_{2}\right), \\
S_{h}^{-1}=\delta\left(z_{1}-h\left(\mathbf{r}_{1}\right)-z_{2}\right) \delta\left(\mathbf{r}_{1}-\mathbf{r}_{2}\right),
\end{gathered}
$$

а оператор растяжения и его обратный:

$$
\begin{array}{r}
S_{\lambda}=\lambda^{1 / 2}\left(\mathbf{r}_{1}\right) \delta\left(\lambda\left(\mathbf{r}_{1}\right) z_{1}-z_{2}\right) \delta\left(\mathbf{r}_{1}-\mathbf{r}_{2}\right), \\
S_{\lambda}^{-1}=\lambda^{-1 / 2}\left(\mathbf{r}_{1}\right) \delta\left(z_{1} / \lambda\left(\mathbf{r}_{1}\right)-z_{2}\right) \delta\left(\mathbf{r}_{1}-\mathbf{r}_{2}\right) .
\end{array}
$$

Действие этих операторов на волновые функции переводит их в

$$
\begin{gathered}
\psi_{h}^{\prime}\left(\mathbf{r}_{2}, z_{2}\right)=S_{h} \psi\left(\mathbf{r}_{1}, z_{1}\right)=\psi\left(\mathbf{r}_{2}, z_{2}-h\left(\mathbf{r}_{2}\right)\right) \\
\psi_{\lambda}^{\prime}\left(\mathbf{r}_{2}, z_{2}\right)=S_{\lambda} \psi\left(\mathbf{r}_{1}, z_{1}\right)=\lambda^{-1 / 2}\left(\mathbf{r}_{2}\right) \psi\left(\mathbf{r}_{2}, z_{2} / \lambda\left(\mathbf{r}_{2}\right)\right) .
\end{gathered}
$$

В новом базисе этих волновых функций (после последовательного применения операторов растяжения и 
сдвига) оператор потенциальной энергии уже не зависит от шероховатости поверхности. Вместо этого к гамильтониану в уравнении (1) в новом базисе добавляется член

$$
V=-\frac{\hbar^{2}}{2 m}\left(S_{\lambda} S_{h} \nabla^{2} S_{h}^{-1} S_{\lambda}^{-1}-\nabla^{2}\right),
$$

который ответственен за рассеяние на шероховатой поверхности. Матричные элементы этого оператора в новом базисе представляются в виде

$$
\left\langle\psi_{m}|V| \psi_{n}\right\rangle=\frac{\hbar^{2} k_{m} k_{n}}{m^{*} S} \int_{S} d^{2} \mathbf{r} h(\mathbf{r}) \exp (-i \mathbf{q r})=\frac{\hbar^{2} k_{m} k_{n}}{m^{*}} f(\mathbf{q}),
$$

где $f(\mathbf{q})=\frac{1}{S} \int_{S} d^{2} \mathbf{r} h(\mathbf{r}) \exp (-i \mathbf{q r})$ - двумерный фурье образ распределения флуктуаций высоты относительно среднего значения $h(\mathbf{r})$ (рельефа поверхности), $k_{m}$ и $k_{n}-z$-компоненты волновых векторов соответственно $m$ и $n-$ подзон размерного квантования в рассматриваемой прямоугольной яме. Присутствие оператора $V$ приводит к тому, что состояние $|\varphi\rangle=\left|k_{n}, \mathbf{k}\right\rangle$ (в импульсном представлении) рассеивается в другие состояния, например $|\psi\rangle=\left|k_{m}, \mathbf{k}^{\prime}\right\rangle$, при этом двумерный вектор рассеяния равен $q(\varphi \rightarrow \psi)=\mathbf{k}^{\prime}-\mathbf{k}$. В результате возможны переходы в пределах одной зоны пространственного квантования $(n=m)$, а также между такими подзонами $(n \neq m)$. Если проекция $\mathbf{q}$ на направление тока (вдоль оси $x$ ) не равна нулю, то такое рассеяние в результате (с учетом всех переходов) приводит к диссипации импульса электрона (тока) и появлению электрического сопротивления. Интенсивность рассеяния вдоль направления $x$ в единицу времени равна

$$
\frac{1}{\tau}=\frac{2 \pi}{\hbar} \sum_{\psi} \frac{-q(\varphi-\psi)}{k_{x}(\varphi)}|(\psi|V| \varphi)|^{2} \delta\left(\varepsilon_{\varphi}-\varepsilon_{\psi}\right) .
$$

Из (6) и (7) следует, что интенсивность рассеяния пропорциональна квадрату модуля двумерного фурье образа рельефа поверхности, т. е. спектральной плотности флуктуаций (СПФ) шероховатости. Удельную проводимость пленки можно найти, используя теорию линейного отклика и подставляя найденный оператор рассеяния $V$, как было сделано в работах $[14,15]$ :

$$
\sigma=\sigma_{0} \frac{3 \pi}{2 d k_{F}} \sum_{n=1}^{N_{d}} \frac{1-\left(k_{n} / k_{F}\right)^{2}}{1+\left(d_{1}^{*} / d\right)^{3} n^{2}}
$$

где $d_{n}^{*}=\left(\pi^{2} Q_{n} l_{0} / k_{F}^{2}\right)^{1 / 3} n^{2 / 3}, n-$ номер подзоны, $Q_{n}=m^{*} d k_{F} /\left(\hbar k_{n}^{2} \tau_{n}\right), l_{0}$ - длина свободного пробега электронов в объеме, $\sigma_{0}$ - проводимость объемного материала. Переходя в (7) к интегрированию по углу рассеяния, находим

$$
\begin{aligned}
Q_{n}= & \frac{2 \hbar^{2} k_{F}^{2}}{d k_{F} m^{*}} \sum_{m=1}^{N d} \int_{0}^{\pi} d \phi\left(1+\frac{\sqrt{k_{F}^{2}-k_{m}^{2}}}{\sqrt{k_{F}^{2}-k_{n}^{2}}} \cos (\phi)\right) \\
& \times \frac{k_{m}^{2}}{k_{F}^{2}}|f(\mathbf{q})|^{2} .
\end{aligned}
$$

Как правило, в моделях рассеяния электронов на поверхности используют гауссову функцию для СПФ. Из такого приближения однозначно следует, что рассеяние электронов с большим $q$ можно пренебречь, и рассеяние является малоугловым для эпитаксиальных пленок с большой корреляционной длиной шероховатости с углом рассеяния $\phi<q_{c} / k_{F}$, где $q_{c}=2 \pi / L_{c}$, $L_{c}$ - корреляционная длина шероховатости, а при $\left(3 / 2 k_{F}\right) \pi^{2} / d^{2} \geq 1 / L_{c}$ реализуется режим только внутриподзонного рассеяния. Однако в модели самоаффинных фракталов [17], которую можно использовать для описания шероховатости поверхностей раздела, ассимптотика $|f|^{2}(q)$ при больших $q$ равна $q^{-2 H}$ с точностью до некоторого численного множителя, где $H$ - показатель шероховатости (связанный с фрактальной размерностью Хауздорфа $D$ соотношением $H=3-D)$. Можно показать, что при $H<3 / 4$ рассеяние малоугловых $(n \sim 1)$ электронов на большой угол существенно и всегда реализует режим межподзонного рассеяния. При этом в формуле (9) сумма по номерам подзон расходится. Это приводит к большим значениям $d_{1}$ в формуле (8) и к квадратичной зависимости $\sigma$ от $d$. В то время как при больших Н множитель при $n^{2}$ в формуле (8) мал, что приводит к более пологим зависимостям проводимости от толщины пленки. Согласно работе [17], зависимость проводимости от толщины может быть и более резкой. Чем больше фрактальная размерность рельефа поверхности пленки, тем зависимость от толщины резче.

\section{2. Методики экспериментов}

В качестве исследуемых образцов для верификации данной модели транспорта электронов изготовлены эпитаксиальные пленки Мо. Для выращивания пленок использовался метод импульсного лазерного осаждения в сверхвысоком вакууме $\left(10^{-9}\right.$ Torr $)$. Подробнее технология выращивания пленок описана в наших предыдущих работах [24-26]. Морфология выращенных пленок исследовалась методами АСМ и СТМ. Для СТМ измерений была использована платиновая игла, для которой предварительно проводилась проверка одноострийности по кривой подвода. Размеры СТМ кадров составлял $300 \times 300 \mathrm{~nm}$, а АСМ кадров - $1000 \times 1000 \mathrm{~nm}$. СТМ измерения выполнены на сканирующем зондовом микроскопе СММ-2000 (Завод ПРОТОН, г. Москва, Россия) на сканере с резонансной частотой $12 \mathrm{kHz}$ [27], а ACM - на микроскопе P47-Solver (НТ-МДТ, Москва, Зеленоград, Россия) с использованием кантилеверов TipsNano (Москва, Зеленоград, Россия). Также было снято тестовое АCM изображение высокого разрешения на СММ-2000, для получения которого использовался кантилевер фирмы Brucker (США) марки „МSCT“ с острием иглы радиусом $2 \mathrm{~nm}$. На СТМ изображениях параметры шероховатости рассчитаны программой микроскопа СММ-2000 в соответствие с международным стандартом ISO 4287, на ACM изображениях эти же параметры были рассчитаны с помощью программы ,gwyddion“" [28]. 


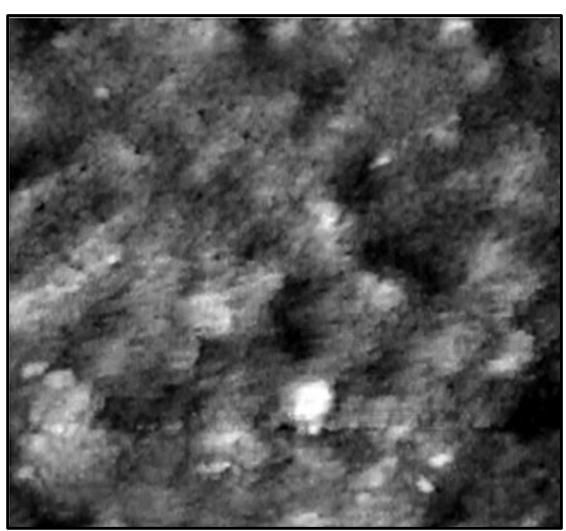

$a$
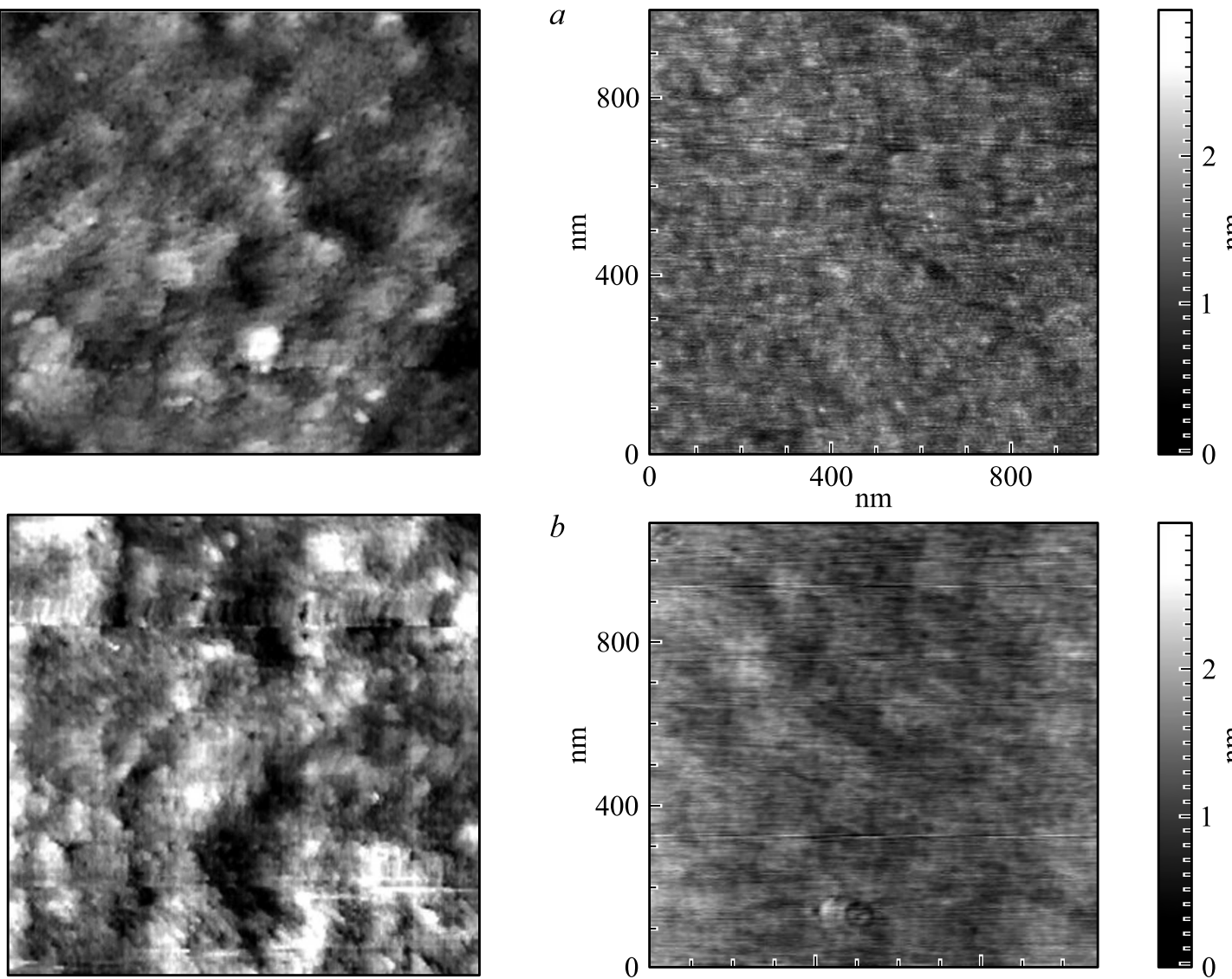

$b$

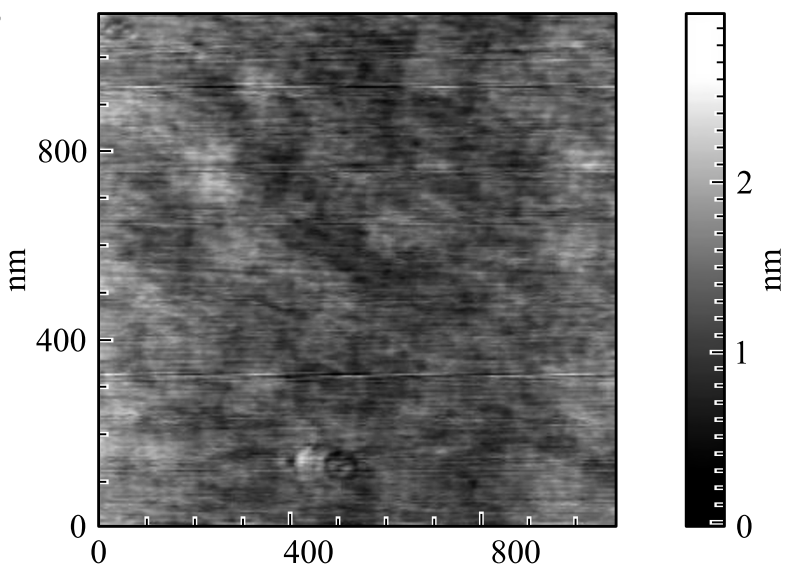

$\Xi$
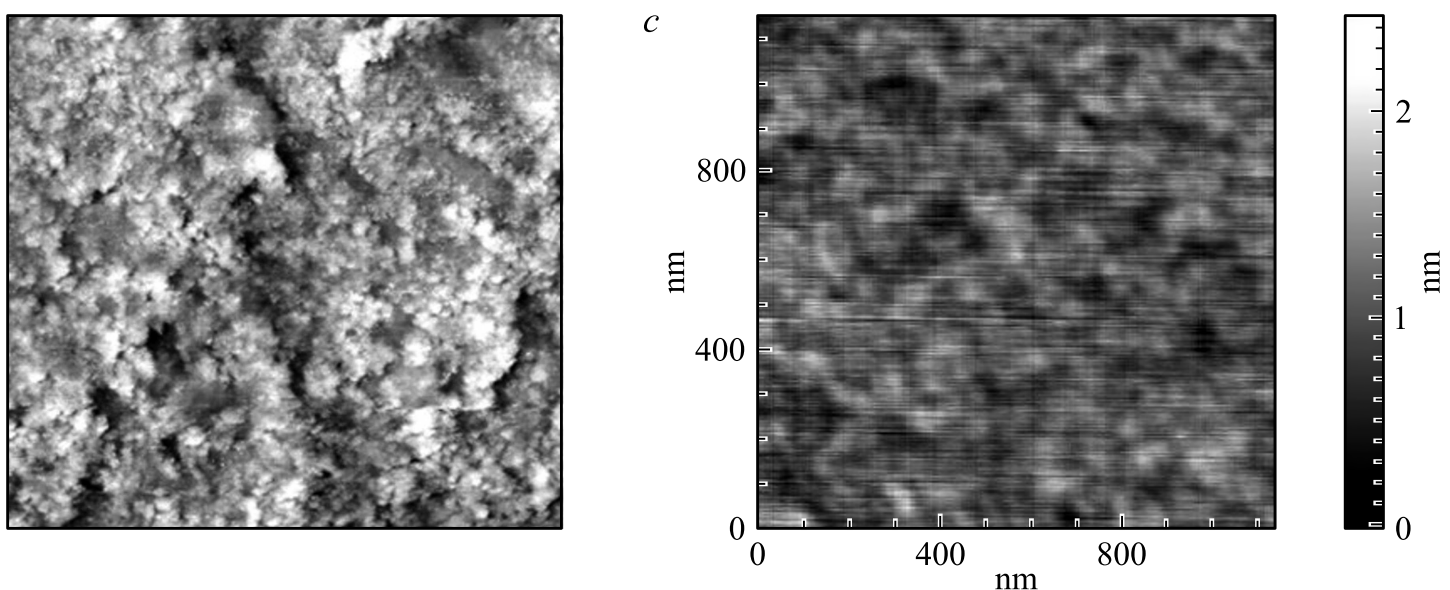

Рис. 1. СТМ (слева) $300 \times 300 \mathrm{~nm}$ и АСМ (справа) $1000 \times 1000 \mathrm{~nm}$ изображения поверхности пленок Мо с толщинами $65(a)$, $55(b)$ и $13(c) \mathrm{nm}$, выращенных на $R$-плоскости сапфира.

\section{3. Сравнение результатов СТМ и АСМ измерений}

На рис. 1 показаны АСМ и СТМ изображения поверхности трех выращенных пленок Мо разной толщины. Из рисунка видно, что АСМ и СТМ измерения дают разные результаты. Полный разброс высот на АСМ-кадре получается всегда меньше, чем на СТМ-кадре. Разрешение в режиме СТМ значительно больше, поскольку более острая СТМ-игла лучше проникает в малые поры, однако пока остается неопределенным вопрос степени влияния неоднородности по проводимости и работе выхода электрона на точность определения высот точек рельефа, что требует дальнейшего более подробного изучения по отношению к элементному и химическому состоянию материалов на поверхности изучаемых нами образцов.

Зависимости среднеквадратичной шероховатости, корреляционной длины и фрактальной размерности от толщины пленок показаны на рис. 2. Среднеквадратичная шероховатость, полученная из СТМ изображений, в 

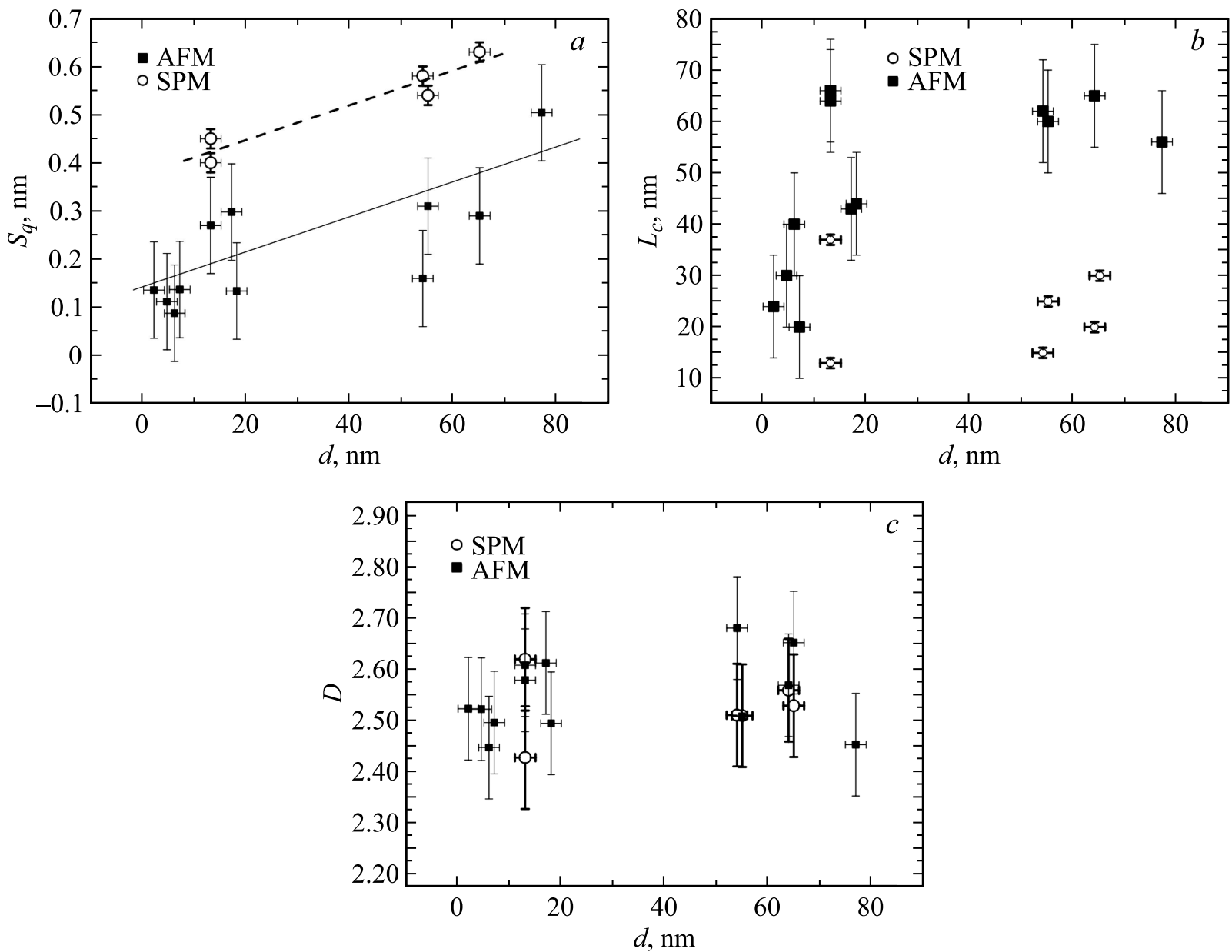

Рис. 2. Зависимость средней шероховатости $(a)$, корреляционной длины $(b)$ и фрактальной размерности $(c)$ поверхности пленок Мо от их толщины по результатам АСМ и СТМ измерений.

основном выше, чем из АСМ изображений одного и того же образца (рис. 2,a). В то же время корреляционная длина, полученная из СТМ, в разы меньше, чем из АСМ (рис. 2,b), а фрактальная размерность, полученная из СТМ, превышает ту, что получена из АСМ (рис. 2,c). Это свидетельствует о том, что подстановка в формулу (8) спектральной плотности флуктуаций, полученную из СТМ, даст большую вероятность рассеяния на большие углы.

\section{4. Зависимость удельной проводимости пленок от их толщины}

Для исследования поведения проводимости пленок при малых толщинах была изготовлена новая серия образцов с толщинами менее $10 \mathrm{~nm}$. Были измерены сопротивления пленок, напыленных через маску в виде мостиков, и получены зависимости их сопротивления от температуры, начиная от комнатной до температуры жидкого гелия $4.2 \mathrm{~K}$ с шагом $0.1 \mathrm{~K}$. Зависимости сопротивления от температуры для ультратонких пленок

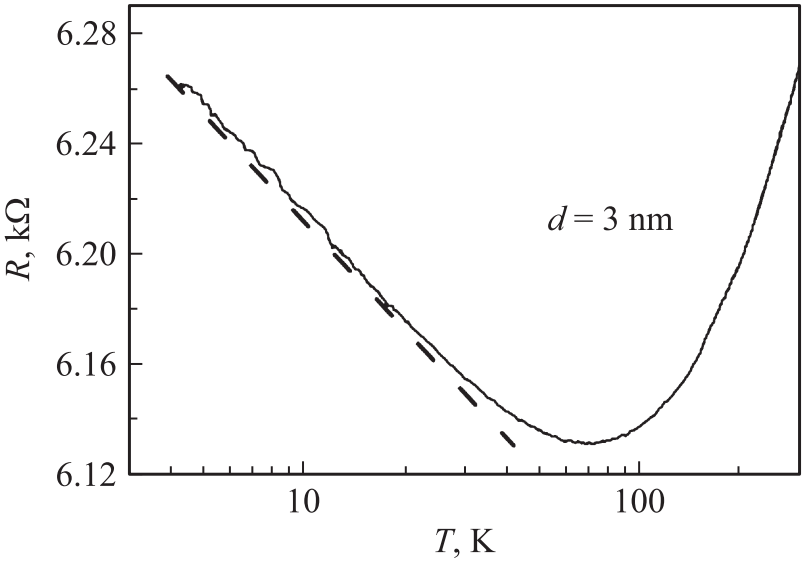

Рис. 3. Температурная зависимость сопротивления эпитаксиальной пленки Мо толщиной менее $3 \mathrm{~nm}$.

имели характерный вид с минимумом при температурах 30-100 K, который обусловлен процессами „вымерзания“ фононов при уменьшении температуры ниже комнатной и появлением эффекта слабой локализации [29] 

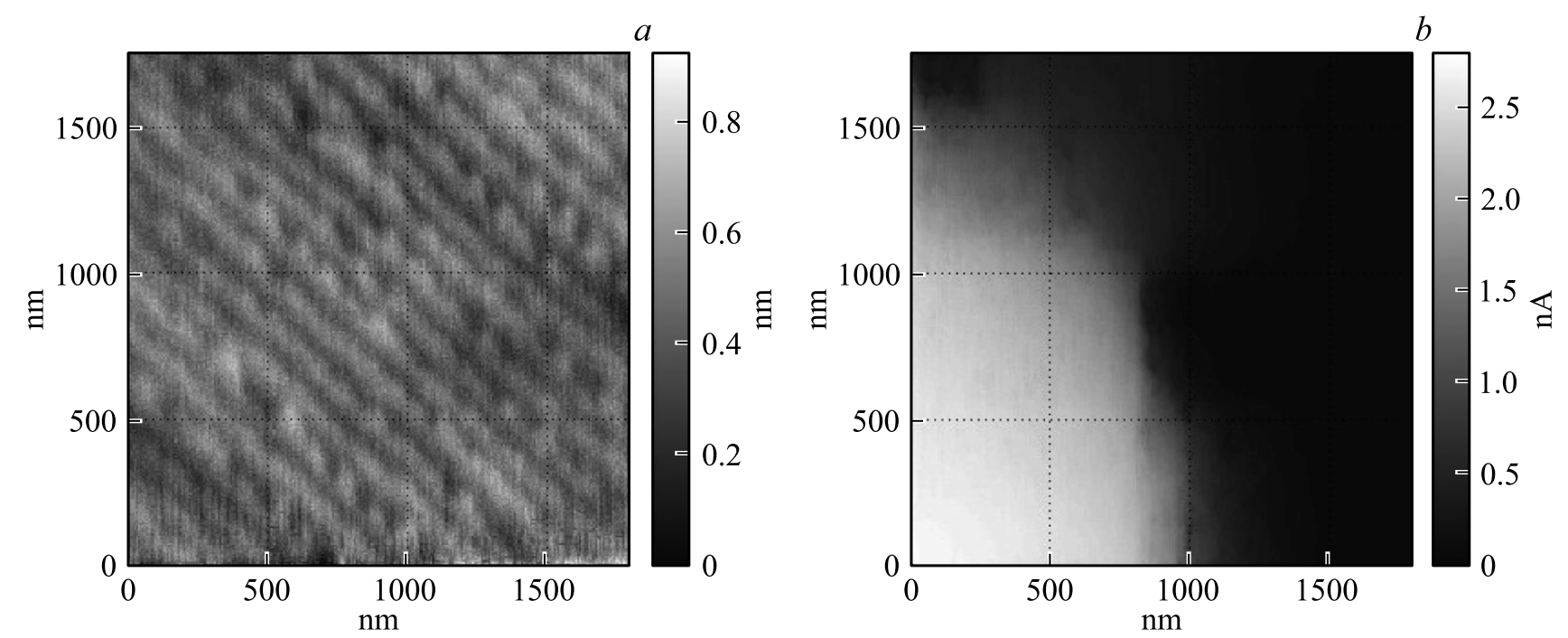

Рис. 4. АСМ изображение $(a)$ и изображение в токах растекания $(b)$ края пленки Мо, толщиной $\sim 6 \mathrm{~nm}$, во время одновременной записи проводящим кантилевером.
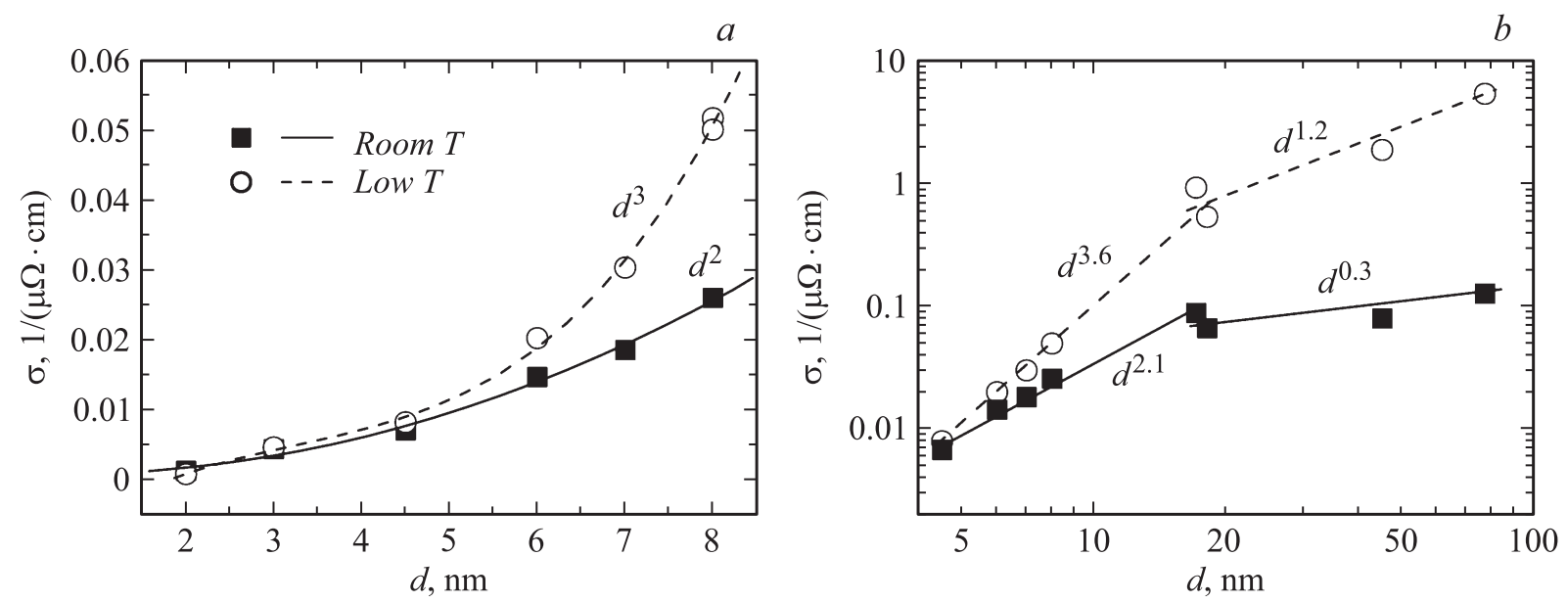

Рис. 5. Зависимость удельной проводимости эпитаксиальных пленок Мо от их толщины при малых толщинах $(a)$ и во всем диапазоне толщин $(b)$. Квадраты — комнатная температура, кружки — точка минимума сопротивления.

при дальнейшем уменьшении температуры. Характерная зависимость сопротивления от температуры для пленки Мо толщиной $3 \mathrm{~nm}$ показана на рис. 3. С уменьшением толщины пленок этот минимум сопротивления смещался ближе к комнатной температуре, и разница в сопротивлении при комнатной температуре и в точке минимума сокращалась, поскольку основной вклад в сопротивление давало рассеяние на шероховатой поверхности. АСМ измерения поверхности ультратонких пленок показали, что их рельеф повторяет рельеф сапфировой подложки. На рис. 4 показаны АCМ изображение (рис. 4, $a$ ), а также АCМ изображение, полученное одновременно в режиме токов растекания (рис. 4, $b$ ) края пленки Мо, толщиной $\sim 6 \mathrm{~nm}$. Для одновременного получения этих изображений использовался проводящий кантилевер. Видно, что рельеф ультратонкой пленки не отличается от рельефа подложки, и край пленки можно определить только по появлению проводимости.
Таким образом, при малых толщинах статистические характеристики поверхности пленок (среднеквадратичная шероховатость, корреляционная длина и фрактальная размерность) не меняются с толщиной и совпадают с характеристиками подложки, которые известны [18]. ACM измерения в режиме токов растекания также показывают, что пленки сплошные (реализуется послойный рост).

Зависимости удельной проводимости пленок от толщины при комнатной температуре и в точке минимума сопротивления представлены на рис. 5. При малых толщинах $(2-8 \mathrm{~nm})$ зависимость удельной проводимости от толщины при комнатной температуре близка к квадратичной, а в точке минимума сопротивления к кубической (рис. 5,a). Квадратичная зависимость подтверждает нашу модель рассеяния электронов на фрактальной поверхности, поскольку показатель шероховатости, согласно экспериментальным данным (рис. 2,c), 
$H=3-D \approx 0.5<3 / 4$. Однако кубическая зависимость проводимости от толщины при низких температурах не объясняется в рамках этой теории. Возможно, ее можно объяснить с точки зрения работы [17]. При этом рассеянием на фононах можно пренебречь по сравнению с поверхностным рассеянием. По всей видимости, то, что при малых толщинах пленок их статистические характеристики не меняются с толщиной, и объясняет достаточно гладкую степенную зависимость на рис. 5, a. При больших толщинах характер зависимости удельной проводимости от толщины меняется. При комнатной температуре она ведет себя как $\sigma \sim d^{0.3}$, а в точке минимума сопротивления - близка к линейной. Такое резкое изменение хода кривых можно объяснить изменением рельефа поверхности, который сам начинает сильно зависеть от толщины (рис. $2, b)$.

\section{5. Обсуждение результатов}

Полученные экспериментальные результаты устраняют противоречие между наблюдаемой квадратичной размерной зависимостью $\sigma$ от толщины при комнатной температуре и большой величиной корреляционной длины по сравнению с фермиевской длиной волны электрона $L_{c} / \lambda_{F} \gg 1$ для исследуемых пленок. Тем не менее при больших толщинах зависимость $\sigma$ от толщины даже более пологая, чем предсказывает теория для гауссового СПФ, что можно объяснить зависимостью рельефа поверхности от толщины. Сравнение результатов СТМ и АСМ измерений показало существенное (в разы) отличие среднеквадратичной шероховатости и корреляционной длины, полученных двумя методами. Вследствие этого имеется необходимость в дальнейших дополнительных исследованиях степени достоверности определения высот рельефа методом СТМ при наличии неоднородностей проводимости и работы выхода электрона по поверхности. При этом в настоящей работе мы пока в большей степени опирались на результаты снятия рельефа методом АСМ.

\section{Заключение}

Методами СТМ и АСМ исследована поверхность эпитаксиальных пленок Мо, выращенных на $R$-плоскости сапфира. Из полученных изображений были найдены статистические характеристики поверхности, от которых зависит сопротивление, вызванное рассеянием на поверхности. Найдены зависимости от толщины удельной проводимости пленок при комнатной температуре и при температуре, где их сопротивление минимально. Было проведено сравнение полученных данных с фрактальной моделью шероховатости поверхности и ее влияния на электронно-транспортные свойства тонких пленок, учитывающей морфологию поверхности через спектральную плотность флуктуаций.

\section{Конфликт интересов}

Авторы заявляют, что у них нет конфликта интересов.

\section{Список литературы}

[1] Kapur P., Mc Vittie J.P., Saraswat K.C. // IEEE Trans. Electron Dev. 2002. Vol. 49. P. 590-597.

[2] Hain M., Kürner H., Neureither B., Röhl S. // Appl. Surf. Sci. 1995. Vol. 91. P. 374-377.

[3] Pan C.Y., Naeemi A. // IEEE Electron Dev. Lett. 2014. Vol. 35. N 2. P. $250-252$.

[4] Fuchs K. // Math. Proc. Cambridge Philos. Soc. 1938. Vol. 34. P. 100.

[5] Sondheimer E.H. // Adv. Phys. 1952. Vol. 1. P. 1.

[6] Mayadas A.F., Shatzkes M. // Phys. Rev. B. 1970. Vol. 1. P. 1382.

[7] Mayadas A.F., Shatzkes M., Janak J.F. // Appl. Phys. Lett. 1969. Vol. 14. P. 345.

[8] Falkovsky L.A. // Adv. Phys. 1983. Vol. 32. P. 753.

[9] Tesanovic Z., Jaric M.V., Maekawa S. // Phys. Rev. Lett. 1986. Vol. 5. P. 2760.

[10] Trivedi N., Ashcroft N.W. // Phys. Rev. B. 1988. Vol. 38. P. 12298.

[11] Makarov N.M., Moroz N.M., Yampol'skii V.A. // Phys. Rev. B. 1995. Vol. 52. P. 6087.

[12] Михайлов Г.М., Маликов И.В., Черных А.В. // Письма в ЖЭТФ. 1997. Т. 66. № 11. С. 693-698. [Mikhailov G.M., Malikov I.V., Chernykh A.V. // JETP Lett. 1997. Vol. 66. N 11. P. 725-731.]

[13] Soffer S.B. // J. Appl. Phys. 1967. Vol. 38. N 4. P. 1710-1713.

[14] Fishman G., Calecki D. // Phys. Rev. B. 1991. Vol. 43. P. 11581-11585.

[15] Sheng L., Xing D.Y., Wang Z.D. // Phys. Rev. B. 1995. Vol. 51. P. 7325 .

[16] Munoz R.C., Finger R.Y., Arenas C.D., Kremer G., Moraga L. // Phys. Rev. B. 2002. Vol. 66. P. 205401.

[17] Palasantzas G. // Phys. Rev. B. 1998. Vol. 58. N 15. P. 9685-9688.

[18] Fischer G., Hoffmann H. // Solid State Commun. 1980. Vol. 35 P. 793.

[19] Hensel J.C., Tung R.T., Poate J.M., Unterwald F.C. // Phys. Rev. Lett. 1985. Vol. 54. P. 1840.

[20] Munoz R.C., Vidal G., Kremer G., Moraga L., Arenas C., Concha A. // J. Phys.: Condens. Matter. 2000. Vol. 12. P. 2903.

[21] Munoz R.C., Vidal G., Mulsow M., Lisoni J.G., Arenas C., Concha A., Mora F., Espejo R., Kremer G., Moraga L., Esparza R., Haberle P. // Phys. Rev. B. 2000. Vol. 62. P. 4686.

[22] Sambles J.R., Elsom K.C., Jarvis J.D. // Philos. Trans. R. Soc. A. 1982. Vol. 304. P. 365.

[23] Фомин Л.А., Маликов И.В., Винниченко В.Ю., Калач К.М., Пяткин С.В., Михайлов Г.М. // Поверхность. 2008. № 2. C. 1-6. [Fomin L.A., Malikov I.V., Vinnichenko V.Yu., Kalach K.M., Pyatkin S.V., Mikhailov G.M. // J. Synch. Investig. 2008. Vol. 2. P. 104-109.]

[24] Malikov I.V., Mikhailov G.M. // J. Appl. Phys. 1997. Vol. 82. N 11. P. 5555-5559.

[25] Mikhailov G.M., Chernykh A.V., Petrashov V.T. // J. Appl. Phys. 1996. Vol. 80. P. 948. 
[26] Михайлов Г.М., Маликов И.В., Черных А.В., Петрашов B.T. // ФТТ. 1996. Т. 38. С. 3212. [Mikhailov G.M., Malikov I.V., Chernykh A.V., Petrashov V.T. // Phys. Solid State. 1996. Vol. 38. P. 1754.]

[27] Логинов Б.А., Логинов П.Б., Логинов В.Б., Логинов А.Б. // Наноиндустрия. 2019. № 6. С. 32-44.

[28] Gwyddion - Free SPM (AFM, SNOM/NSOM, STM, MFM) data analysis software, http://gwyddion.net/, дата последнего обращения 5.03.2020.

[29] Bergman G. // Phys. Rep. 1984. Vol. 107. N 1. P. 1-58. 